\title{
Statistical analysis and application of competing risks model with regression
}

\author{
Petr Volf * \\ Institute of Information Theory and Automation, AS CR \\ Pod vodarenskou vezi 4, Prague 8, Czech Republic \\ E-mail: 〈volf@utia.cas.cz〉
}

\begin{abstract}
The paper deals with the methods of statistical analysis of dependent competing risks in the presence of covariates. The problem of identification of marginal and joint distributions of competing random variables is recalled and certain identifiability results in the framework of regression models are presented. The main objective is then to study the case when the correlation of competing variables depends on covariates, as this phenomenon has not been taken into account in the most of papers dealing with the identifiability of competing risks models with regression. Such a dependence is demonstrated and estimated on a real example with unemployment data.
\end{abstract}

Keywords: competing risks, copula, Cox's regression, survival analysis, unemployment

Received: September 21, 2018; accepted: March 19, 2019; available online: July 4, 2019

DOI: 10.17535 /crorr.2019.0002

\section{Introduction}

The case of mutually dependent competing risks often appears in the field of reliability, biostatistics and medical studies, as well as in demography, labor statistics, insurance, and in econometrics generally. The interest in the problem dates back to 70-ties of the last century. It is well known that, in general, without additional assumptions (e.g. on a parametric form of distributions) the model is not identifiable. As nicely shown in Tsiatis [13], for each level of dependence we may obtain different estimate of joint probability distribution of both random times, and we are not able to distinguish among them. Further, Heckman and Honoré [6] showed (rather than proved, as their argumentation is more verbal than precisely mathematical) that when an additional information on covariates is available, identification is possible. Namely, they dealt with competing times with Cox's or with AFT regression models. Their result is in the background of the unemployment duration study by Han and Hausman [5], with two competing events of unemployment termination. However, they quite neglected the possibility that the parameter characterizing mutual dependence of risks (e.g. their correlation) may also depend on covariates. Then the problem of non-identifiability arises anew. Later on the case of competing risks with covariates was studied by many other authors, in a more precise way, already with the aid of a copula describing the dependence, see e.g. Lee [9], Berg et al. [2]. However, the problem of possible dependence of copula parameter on the covariates was not discussed. That is why the present paper opens this problem.

The paper has the following parts: First, the scheme of competing risks is introduced and the methods and problems of its statistical analysis reminded, namely the problem of possible non-identifiability of their marginal probability distributions is given in Section 2. We shall

${ }^{*}$ Corresponding author. 
mention also certain identifiability results in the framework of regression models. Then the notion of copula is recalled and used in competing risks model formulation. In Section 3 the Gauss copula is introduced and the likelihood function is derived. It is then use as a basis for the Bayes solution of the parameters estimation problem. Finally, in Section 4, the Gauss copula and Cox's regression model are jointly applied to a real example. We use the data of Kadane and Woodworth [8] stored in the Statlib database. The data record two competing terminations of employment in certain company. It will be shown that the model parameters and, in particular, the correlation of the risks, depend on considered covariates. The model will be fully parametric, numerical solution will consist in a randomized search for parameters estimates with the aid of the Metropolis-Hastings MCMC algorithm in the Bayes framework.

\section{Competing risks and non-identifiability problem}

Let us imagine that a certain event (e.g. a failure of a device) can be caused by $\mathrm{K}$ reasons. Formally, there are $K$ (possibly dependent) random variables, times $T_{j}, j=1, \ldots, K$, running simultaneously. Observation is terminated at minimum of them. Sometimes there is another random or deterministic variable $C$ of right censoring assumed to be independent of all $T_{j}$. Standardly, $C$ may cause the termination of observation from "external" reasons, before any of expected events occur; in this case $C<T_{j}$ for all $j$. We assume that all variables $T_{j}$ are of continuous type. Let $\bar{F}_{K}\left(t_{1}, \ldots, t_{K}\right)=P\left(T_{1}>t_{1}, \ldots, T_{K}>t_{K}\right)$ be the joint survival function of $\left\{T_{j}\right\}$. However, instead the 'net' survivals $T_{j}$ we observe just 'crude' data (sometimes called also 'the identified minimum') $Z=\min \left(T_{1}, \ldots, T_{K}, C\right)$ and the indicator $\delta=j$ if $Z=T_{j}, \delta=0$ if $Z=C$. Such data lead us to direct estimation of the distribution of $Z^{*}=\min \left(T_{1}, \ldots, T_{K}\right)$, for instance its survival function $S(t)=P\left(Z^{*}>t\right)=\bar{F}_{K}(t, \ldots, t)$. Further, we can estimate so called incidence densities

$$
f_{j}^{*}(t)=d P\left(Z^{*}=t, \delta=j\right)=-\frac{\partial \bar{F}_{K}\left(t_{1}, \ldots, t_{K}\right)}{\partial t_{j}} \mid\left(t_{1}=\ldots=t_{K}=t\right),
$$

and also their integrals, cumulative incidence functions

$$
F_{j}^{*}(t)=\int_{0}^{t} f_{j}^{*}(s) d s=P\left(Z^{*} \leq t, \delta=j\right)
$$

Notice that $\lim F_{j}^{*}(t)=P(\delta=j)<1$ if $t \rightarrow \infty, S(t)=1-\sum_{j=1}^{K} F_{j}^{*}(t)$. Further, so called cause-specific hazard functions for events $j=1,2, \ldots, K$ are defined as

$$
h_{j}^{*}(t)=\lim _{d \rightarrow 0} \frac{P\left(t \leq Z^{*}<t+d, \delta=j \mid Z^{*} \geq t\right)}{d}
$$

and are estimable as well.

However, in general, from data $\left(Z_{i}, \delta_{i}\right), i=1, \ldots, N$ it is not possible to identify the marginal or joint distributions of $\left\{T_{j}\right\}$. Tsiatis in [13] has shown that for arbitrary joint model we can find a model with independent components having the same incidences, i.e. we cannot distinguish among the models. Namely, this 'independent' model is given by cause-specific hazard functions $h_{j}^{*}(t)$. It follows that it is necessary to make certain functional assumptions about the form of both marginal and joint distribution in order to identify them. Several such cases are specified for instance in Basu and Ghosh [1]. More recent results on identifiability can be found for example in Escarela and Carriere [3] considering Frank copula and parametric models. Schwarz et al in [12] deal with non-parametric setting and study the inverse problem how from marginal distributions the joint distribution can be estimated, under a given copula class assumption.

Many authors have studied the role of additional information obtained from covariates in the framework of a regression model for examined random times. The results determining conditions 
for full identifiability of such a regression model start from already mentioned Heckman and Honoré [6]. Lee in [9] investigated more general transformation models of regression. A common identifiability assumption consists in sufficiently rich structure of covariates. However, all these studies rely on the supposition that the dependence structure (in copula models given by the copula parameter) does not change with covariates. And this rather strong supposition is therefore the subject of our examination.

\section{Copulas in models for competing risks}

Let us consider, in the sequel, just two competing events, with corresponding random times $S, T$ (eventually together with a right-censoring variable $C$ ). The data are then given as realizations of $N$ i.i.d. couples of random variables $Z_{i}=\min \left(S_{i}, T_{i}, C_{i}\right), \delta_{i}=1,2,0, i=1,2, \ldots, N$. The notion of copula (see e.g. Nelsen [11]) offers a way how to model the joint distribution function $F_{2}(s, t)$ of $S, T$ :

$$
F_{2}(s, t)=\boldsymbol{C}\left(F_{S}(s), F_{T}(t), \theta\right)
$$

where $F_{S}, F_{T}$ are marginal distribution functions of $S, T, \boldsymbol{C}(u, v, \theta)$ is a copula, i.e. a twodimensional distribution function on $[0,1]^{2}$, with uniformly on $[0,1]$ distributed marginal components $U, V . \theta$ denotes the copula parameter which is connected uniquely with correlation of $U, V$, hence also with correlation of $S, T$. It is seen that the use of copula allows to model the dependence structure separately from the analysis of marginal distributions. Hence, the identifiability of the copula (and its parameter) and marginal distributions of $S, T$ can be considered as two separate steps.

Zheng and Klein in [14] have proved that when the copula is known, the marginal distributions are estimable consistently (and then the joint distribution, too, from (4)), even in a non-parametric (so that quite general) setting. However, in general, the knowledge of $\theta$ is needed, because (again due to [13]) without fully determined copula we are not able to distinguish between the 'true' model and corresponding independent one. On the other hand, Zheng and Klein [14] argued that the selection of copula type is not crucial. They also showed it on a simulated example. Later on, the problem of proper copula choice has been analyzed in a set of papers, let us mention here Kaishev et al [7] comparing performance of several copula types. A common agreement is that the knowledge (or a good estimate) of parameter $\theta$ is much more important for correct model of joint distribution, than the copula class selection.

As a consequence, because the knowledge of copula type is still an unrealistic supposition, we can try to use certain sufficiently flexible class of copulas, as approximation, and concentrate to reliable estimation of its parameter. There exist a large number of different copula functions, among them for instance the set of Archimedean copulas. We shall use another rather universal and flexible copula type, namely the Gauss copula.

Let us note that the copula can be used also as a survival copula connecting marginal survival functions $\bar{F}_{S}, \bar{F}_{T}$ of variables $S, T$ to their joint survival function:

$$
\bar{F}_{2}(s, t)=\boldsymbol{C}\left(\bar{F}_{S}(s), \bar{F}_{T}(t)\right)
$$

\subsection{Gauss copula}

Let $X, Y$ be the standard normal random variables $\sim N(0,1)$ tied with (Pearson) correlation $\rho=\rho(X, Y)$. Let us denote by $\varphi(x), \phi(x)$ the univariate standard normal density and distribution function, further by $\phi_{2}(x, y)$ distribution function and by $\varphi_{2}(x, y)$ density function of two-dimensional Gauss distribution with both expectations equal zero and covariance matrix $\Sigma=[1, \rho ; \rho, 1]$. If we define $U=\phi(X), V=\phi(Y)$, we obtain that the couple $(U, V)$ has the 2-dimensional copula distribution on $(0,1)^{2}$ with distribution function

$$
\boldsymbol{C}(u, v)=\phi_{2}\left(\phi^{-1}(u), \phi^{-1}(v)\right)
$$


Naturally, $\rho(U, V) \neq \rho(X, Y)$, while Spearman's correlation coincide, as it is based just on the order statistics. Namely $\rho_{\mathrm{SP}}(X, Y)=\rho_{\mathrm{SP}}(U, V)=\rho(U, V)$, for Gauss and other elliptical copulas it holds that $\rho(X, Y)=2 \cdot \sin \left(\frac{\pi}{6} \rho(U, V)\right)$.

However, our aim is to model the dependence of competing variables $S, T$. Let us assume that their joint survival function is given by Gauss copula (6) used as survival copula:

$$
\bar{F}_{2}(s, t)=\phi_{2}\left(\phi^{-1}\left(\bar{F}_{S}(s)\right), \phi^{-1}\left(\bar{F}_{T}(t)\right)\right)
$$

It means that potential random variables forming the Gauss copula (6) are $U=\bar{F}_{T}(T), V=$ $\bar{F}_{S}(S)$ and $X=\phi^{-1}(U), Y=\phi^{-1}(V)$, the only one parameter describing the dependence of $S$ and $T$ in model $(7)$ is $\rho=\rho(X, Y)$ of $(6)$. Though $\rho(S, T)$ differs from it, all values $\rho(S, T)$ can be achieved by a convenient choice of parameter $\rho$. Again $\rho_{\mathrm{SP}}(S, T)=\rho_{\mathrm{SP}}(U, V)$, Let us remark here that the real dependence among $S, T$ can be different, given by another copula type, nevertheless we may conjecture again that the use of Gauss copula offers at least a good and flexible approximation. Thus, we have to concentrate to the estimation of proper correlation value as well as to estimation of marginal distributions of $S$ and $T$.

\subsection{Estimation in Gauss copula model}

When parameter $\rho$ is known, copula (6) is fully defined and from Zheng, Klein [14] it follows that the distribution of $(S, T)$ can be estimated, even in a nonparametric way. On the other hand, without knowledge of $\rho$ nonparametric model is not identifiable and in the parametric setting explicit proofs of identifiability are available for just certain types of marginal distributions specified for instance already in Basu and Ghosh [1]. We shall deal with a richer model including the covariates, and, similarly like Han and Hausman in [5], with the Cox's model of dependence on them. Let us first formulate the maximum likelihood method. The data are $\left(Z_{i}, \delta_{i}\right), i=$ $1, \ldots, N$, the likelihood function then has the form

$$
L=\prod_{i=1}^{N}\left\{-\frac{\partial}{\partial s} \bar{F}_{2}(s, t)\right\}^{I\left[\delta_{i}=1\right]} \cdot\left\{-\frac{\partial}{\partial t} \bar{F}_{2}(s, t)\right\}^{I\left[\delta_{i}=2\right]} \cdot \bar{F}_{2}(s, t)^{I\left[\delta_{i}=0\right]}
$$

evaluated at $s=t=Z_{i}$, with $\bar{F}_{2}(s, t)=P(S>s, T>t)$. From (7) it follows that, for Gauss survival copula, $\bar{F}_{2}(s, t)=\phi_{2}(x, y)$ with $x=\phi^{-1}\left(\bar{F}_{S}(s)\right), y=\phi^{-1}\left(\bar{F}_{T}(t)\right)$. After some computation, we obtain that

$$
\begin{aligned}
L=\prod_{i=1}^{N}\left\{f_{S}\left(Z_{i}\right) \phi_{1}\left(Y_{i} ; \rho X_{i}, 1-\rho^{2}\right)\right\}^{I\left[\delta_{i}=1\right]} & \\
& \cdot\left\{f_{T}\left(Z_{i}\right) \phi_{1}\left(X_{i} ; \rho Y_{i}, 1-\rho^{2}\right)\right\}^{I\left[\delta_{i}=2\right]} \cdot \phi_{2}\left(X_{i}, Y_{i}\right)^{I\left[\delta_{i}=0\right]},
\end{aligned}
$$

again with $X_{i}=\phi^{-1}\left(\bar{F}_{S}\left(Z_{i}\right)\right), Y_{i}=\phi^{-1}\left(\bar{F}_{T}\left(Z_{i}\right)\right)$. Here $\phi_{1}\left(x ; \mu, \sigma^{2}\right)$ denotes the distribution function of one-dimensional normal distribution $N\left(\mu, \sigma^{2}\right)$, evaluated at $x$. Parameter $\rho$ is hidden in $\phi_{1}$ and in $\phi_{2}$. Distributions of $S$ and $T$ are present both explicitly and also implicitly, in transformed $X_{i}, Y_{i}$. It is seen that the problem of maximization may be a difficult optimization task and has to be solved by a convenient numerical procedure. In the following real data example the model is fully parametric. The search for optimal estimates of parameters is performed with the aid of the MCMC method, in the Bayes approach framework starting from conveniently chosen uniform priors (c.f. Gamerman [4]). The method generates a representation of Bayes posterior distribution. From it, its sample median will be taken as the point parameter estimate, its sample quantiles allow also to obtain Bayes credibility intervals for parameters. 


\section{Real data example}

The data are available in the StatLib database [15], the "Case K" data. The data appear also in Kadane and Woodworth [8]. There the authors did not use the scheme of competing risks, the aim of their study was to explore whether older employees were or not "discriminated" having higher rate of dismissal. The problem is that the risk of dismissal is correlated with the risk of voluntary leave, and that these risks can compensate each other. However, in fact, the question of possible discrimination is behind our analysis, too, though just implicitly.

The data contain the records of all persons employed by a firm during the period of observation, from 1.1.1900 to 31.1.1995, namely their dates of birth, dates when persons were hired by the company and when they left it, either voluntarily or were forced to leave (dismissed). There were together 412 people, from them 96 were dismissed, 108 left voluntarily, the rest, 208 employees, were still with the company at the end of data collection period. The initial state was 163 employees, hence 249 persons joined the company during the followed period, thus changing the "risk set" of the study. From them, 56 were dismissed, 81 left voluntarily, 112 stayed till the end of study.
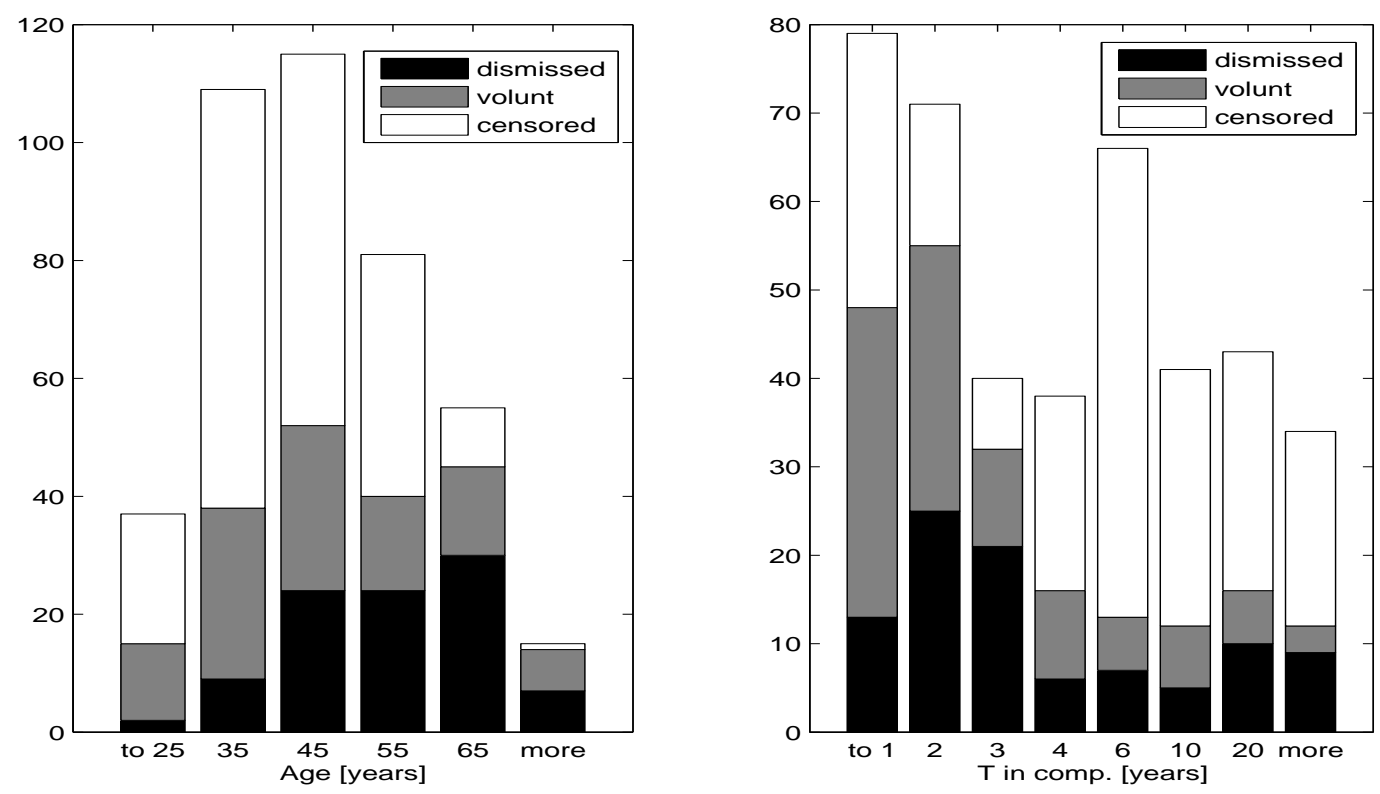

Figure 1: Distribution of modes of job termination versus age intervals (left) and lengths of stay with company (right)

Hence, we deal with two competing risks of the employment termination, and we assume that the risks are dependent. The time considered is the calendar time, in days, from 1 to 1857, the end of study is also the fixed time of censoring, namely $C=1857$ is the upper bound for each personal record (it is so called type I censoring by fixed value). It is expected that the development of the company can be the reason for changing rates of leaving it. 

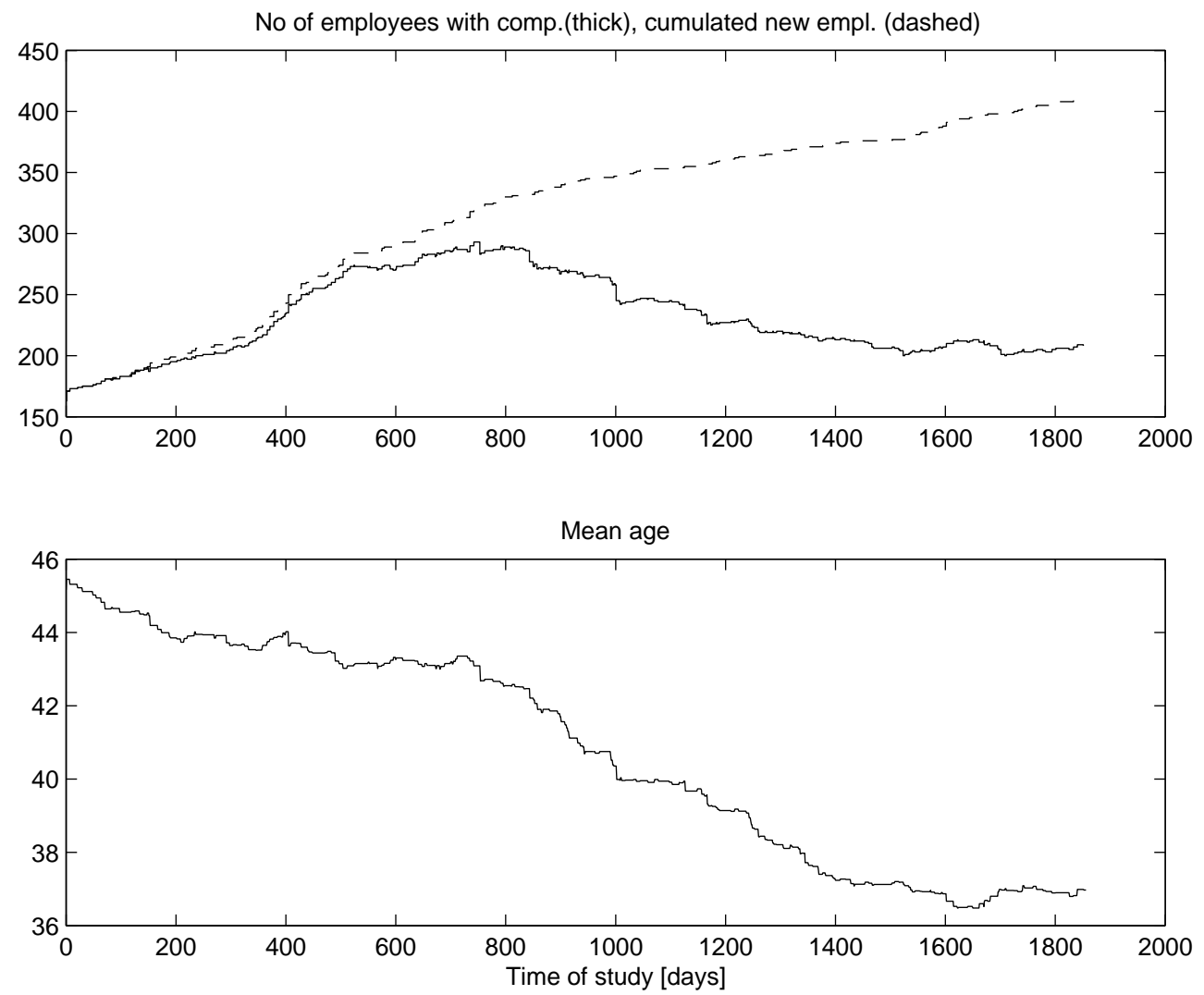

Figure 2: Development of number of employees during the study period (above). Dashed curve shows the increase of newly hired people, thick curve then actual number of employees. The lower plot shows the development of average age of employees

The changes in "personal policy" of the company can be traced already from Figure 1, upper plot. The period of higher intensity of employees fluctuation (which could be taken as an indicator of certain non-stable conditions in the firm) started at about day 800 and lasted almost another 800 days. The age (in years) of employees at the moment of leave or censoring, as well as the time spent working in the company were taken as covariates, because it was expected that both could influence the decision and were changing the relation between the rate of compulsory and voluntary leavings. The age varied from 19 to 72 years, its median was 40. Time spent at the company varied from only 11 days to more than 41 years (15086 days), with median 1322 days. Figure 2 shows variation of all 3 events (dismissal, voluntary leave, censoring) in different groups of age and of time with company. From these graphs it could be further seen that dismissals were more frequent in groups of higher age. On the contrary, the rate of voluntary leaves increased in the last period of the study, in particular concerning younger employees not being with the company long. 


\subsection{Competing risks with Cox's regression model}

Both random times are described by their hazard rates

$$
h_{j}(t ; \boldsymbol{x})=h_{0 j}(t) \cdot \exp \left(\beta_{j} \cdot a g e\right) \cdot \exp \left(\gamma_{j} \cdot t c\right),
$$

where $j=1,2$ for related risks of dismissal and voluntary leave, respectively. Covariates $\boldsymbol{x}=(a g e, t c)$ are the age of persons and the time spent with company, at the moment of job termination or of censoring. Further, $h_{0 j}(t)$ are baseline hazard rates, $\beta_{j}$ are regression parameters of dependence on age, while $\gamma_{j}$ describe dependence on the time spent in company, "tc". Finally, $t$ is the time of the study in days. Further, to make computation easier, we assume that the baseline distributions correspond to Weibull ones. The baseline hazard rates and their cumulated (integrated) versions have then the form

$$
h_{0 j}(t)=\frac{b_{j} \cdot t^{b_{j}-1}}{a_{j}^{b_{j}}}, H_{0 j}(t)=\left(\frac{t}{a_{j}}\right)^{b_{j}},
$$

where $a_{j}, b_{j}, j=1,2$ are Weibull scale and shape parameters. When the Cox's regression term

is added, the distribution of both random times for given covariate values is still Weibull, with the same shape parameters $b_{j}$ and scale parameters depending on covariates, namely

$$
a_{j}(\boldsymbol{x})=a_{j} \exp \left(-\frac{\beta_{j}}{b_{j}} a g e\right) \exp \left(-\frac{\gamma_{j}}{b_{j}} t c\right)
$$

The complete model then contains 9 unknown parameters, $a_{j}, b_{j}, \beta_{j}, \gamma_{j}$ for $j=1,2$, and $\rho$ controlling the dependence of both competing risks via the Gauss copula.

In order to show the dependence of correlation of random times of both competing events on considered covariates, the sample was divided into four sub-samples: age $\leq 40$ or $>40$ (years), time with company $\leq 1322$, > 1322 (days). Each sub-sample was analyzed separately. Naturally, covariates age and $t c$ were also mutually dependent, however not strongly, with correlation approximately 0.3 .

\subsection{Results}

The model is fully parametric, however already from the form of the likelihood function (4) it is seen that the computation may be difficult. Therefore the estimates of parameters were obtained with the aid of a random search using the MCMC method, namely the Metropolis-Hastings algorithm. Prior distributions of parameters were chosen uniform in reasonable intervals, the first rough estimate was obtained under the assumption of no dependence, i.e. $\rho=0$. Then, naturally, the value of parameter $\rho$ was alternated, too. Each computation used 20000 iterations, the last half from them were taken as yielding a representation of posterior distribution. Then sample medians were taken as parameters estimates, while corresponding sample quantiles as borders of credibility intervals.

\begin{tabular}{|l|rr|}
\hline & $\mathrm{tc} \leq 1322$ & $\mathrm{tc}>1322$ \\
\hline age $\leq 40$ & 0.6891 & 0.7814 \\
quantiles & $(0.2024,0.8673)$ & $(0.2504,0.8823)$ \\
$n_{1}, n_{2}, n_{0}$ & $15,47,54$ & $4,11,83$ \\
\hline age $>40$ & -0.5850 & 0.1948 \\
quantiles & $(-0.7140,-0.3559)$ & $(0.0083,0.3970)$ \\
$n_{1}, n_{2}, n_{0}$ & $46,34,10$ & $31,16,61$ \\
\hline
\end{tabular}

Table 1: Sample medians and 90\% credibility intervals, i.e. 5\% and 95\% sample quantiles (in brackets) of estimated $\rho$ in 4 different groups; $n_{1}, n_{2}, n_{0}$ are numbers of people dismissed, left voluntarily, censored in each group 
The results are collected in Table 1. It is seen that estimated correlations differ among groups, though a statistically significant difference is just between the result for age $>40, t c \leq$ 1322 and the rest (age in years, "tc" in 100 days). Nevertheless, estimated correlation depends on both covariates. The interpretation of higher positive correlation in the group of younger employees could be that they are more flexible and in the case of symptoms of approaching negative changes in the company they are more prone to search for a new employment.

On the other hand, negative correlation means that when the (potential) rate of one event increases, the rate of the second decreases. It could be interpreted, in the case of increasing risk of being dismissed, like passive waiting, without own decision. Notice also that some groups contain just small number of noncensored observations, leading to weak confidence of estimates, here represented by rather wide credibility intervals.

\begin{tabular}{|r|rrrr|}
\hline & age $\leq 40, \mathrm{tc} \leq 1322$ & age $>40, \mathrm{tc} \leq 1322$ & age $\leq 40, \mathrm{tc}>1322$ & age $>40, \mathrm{tc}>1322$ \\
\hline$\beta_{1}$ & 0.0155 & 0.0015 & 0.0348 & 0.1176 \\
& $(0.0088,0.0627)$ & $(-0.0003,0.0220)$ & $(-0.0253,0.0811)$ & $(0.1026,0.1761)$ \\
\hline$\beta_{2}$ & -0.2438 & 0.0028 & -0.0029 & 0.0037 \\
& $(-0.2937,-0.1947)$ & $(-0.0009,0.0255)$ & $(-0.0207,0.0164)$ & $(-0.0021,0.0053)$ \\
\hline$\gamma_{1}$ & 0.0003 & 0.0061 & 0.1175 & 0.0985 \\
& $(-0.0008,0.0015)$ & $(-0.0038,0.0212)$ & $(0.0759,0.1621)$ & $(0.0758,0.1183)$ \\
\hline$\gamma_{2}$ & -0.2526 & -0.3553 & -0.061 & -0.0023 \\
& $(-0.3065,0.2124)$ & $(-0.4155,0.2972)$ & $(-0.0879,-0.0387)$ & $(-0.0053,0.0019)$ \\
\hline
\end{tabular}

Table 2: Sample medians and 90\% credibility intervals, i.e. $5 \%$ and $95 \%$ sample quantiles (in brackets) of estimated Cox's regression parameters in 4 different groups

Further, from Table 2 it is seen that also Cox's model parameters characterizing the dependence of hazard rates on covariates differ among groups, and in some groups significantly from the rest (credibility intervals are disjoint). Let us recall that $\beta_{1}$ shows the dependence of risk of dismissal on age, $\beta_{2}$ the dependence of rate of voluntary leave on age, $\gamma_{1}, \gamma_{2}$ the dependence of the same rates on the time with company. For instance, from positive $\beta_{1}$ we can deduce that the risk of dismissal increases significantly with age, in particular for longer employed people. In fact, it could be understood as an indicator of existing discrimination of older employees in the sense of the study [8] of Kadane and Woodworth. Further, younger and just recently engaged employees have larger tendency to leave (negative $\beta_{2}$ as well as negative $\gamma_{2}$ ), while $\gamma_{1}$ is positive, indicating a higher rate of dismissal, for people being with company for a longer time.

As the Cox's model parameters differ among groups (sometimes statistically significantly), we can doubt about sufficient flexibility of a global Cox's model. Actually, one of goodness-of-fit test methods is based on such an approach that the model estimated from all the data is tested on data sub-samples.

\section{Conclusion}

We have studied the problem of competing risks with regression, with the focus on assessing mutual dependence of competing random variables. The joint distribution was expressed with the aid of Gauss copula, while the Cox's regression model described the covariate influence. The model was utilized in an example with real unemployment data. Statistical analysis revealed prevailingly positive correlation between times to both competing events, and also the dependence of correlation on the covariate. This was, in fact, the main purpose of the study.

It has to be said that the Weibull distribution used in order to simplify computations is not an optimal model of actual baseline hazard rates. Better possibilities consist in using a more 
general model, e.g. a mixture of 2 or 3 Weibull hazard rates or a general nonparametric form of baseline rates. However, such a generalization then leads to increased computation problems. The experience with present and similar computational procedures indicates that the likelihood function is, as a rule, rather flat, the convergence of computations to stable estimates is slow, resulting credibility intervals are then quite wide. This phenomenon does not depend on the copula choice, it is a consequence of complicated model structure.

\section{Acknowledgement}

The research was supported by the grant No.18-02739S of the Grant Agency of the Czech Republic.

\section{References}

[1] Basu, A. P. and Ghosh, J. K. (1978). Identifiability of the multinormal and other distributions under competing risks model. Journal of Multivariate Analysis, 8(3), 413-429. doi: 10.1016/0047259x(78)90064-7

[2] Van den Berg, G. J., van Lomwel, A. G. C. and van Ours, J. C. (2008). Nonparametric estimation of a dependent competing risks model for unemployment durations. Empirical Economics, 34(3), 477-491. doi: 10.1007/s00181-007-0131-8

[3] Escarela, G. and Carriere, J. F. (2003). Fitting competing risks with an assumed copula. Statistical Methods in Medical Research, 12(4), 333-349. doi: 10.1191/0962280203sm335ra

[4] Gamerman, D. (1997). Markov Chain Monte Carlo. New York: Chapman and Hall.

[5] Han, A. and Hausman J. A. (1990). Flexible parametric estimation of duration and competing risk models. Journal of Applied Econometrics, 5(1), 1-28. doi: 10.1002/jae.3950050102

[6] Heckman, J. J. and Honoré, B. E. (1989). The identifiability of the competing risks model. Biometrika, 76(2), 325-330. doi: 10.1093/biomet/76.2.325

[7] Kaishev, V. K., Dimitrova, D. S. and Haberman, S. (2007). Modelling the joint distribution of competing risks survival times using copula functions. Insurance: Mathematics and Economics, 41(3), 339-361. doi: 10.1016/j.insmatheco.2006.11.006

[8] Kadane, J. B. and Woodworth, G. G. (2004). Hierarchical models for employment decisions. Journal of Business and Economic Statistics, 22(2), 182-193. doi: 10.1198/073500104000000073

[9] Lee, S. (2006). Identifcation of a competing risks model with unknown transformations of latent failure times. Biometrika, 93(4), 996-1002. doi: 10.1093/biomet/93.4.996

[10] Lin, D. Y. (1997). Non-parametric inference for cumulative incidence functions in competing risks studies. Statistics in Medicine, 16(8), 901-910. doi: 10.1002/(SICI)10970258(19970430)16:8\%3C901::AID-SIM543\%3E3.0.CO;2-M

[11] Nelsen, R. B. (1999). An Introduction to Copulas (Lecture Notes in Statistics). New York: Springer. doi: 10.1007/978-1-4757-3076-0

[12] Schwarz, M., Jongbloed, G. and Van Keilegom, I. (2013). On the identifiability of copulas in bivariate competing risks models. Canadian Journal of Statistics, 41(2), 291-303. doi: 10.1002/cjs.11179

[13] Tsiatis, A. (1975). A nonidentifiability aspects of the problem of competing risks. Proceedings of the National Academy of Sciences, 72(1), 20-22. doi: 10.1073/pnas.72.1.20

[14] Zheng, M. and Klein, J. P. (1995). Estimates of marginal survival for dependent competing risks based on an assumed copula. Biometrika, 82(1), 127-138. doi: 10.1093/biomet/82.1.127

[15] StatLib - Datasets Archive, http://lib.stat.cmu.edu/datasets/caseK.txt [Accessed 01/07/2018]. 